\title{
DETECTION OF FALSIFICATION OF HORILKA BY ADMITTANCE SPECTROSCOPY
}

\author{
Yevhen Pokhodylo, Dr. Sc., Prof., Viktor Kuts, PhD, As.-Prof.; Yuriy Stasyshyn, PhD student \\ Lviv Polytechnic National University,Ukraine, e-mail:viktor kuts@ukr.net
}

\begin{abstract}
The methods of quality control of horilka by electrical parameters, in particular, dielectric constant, specific conductivity, and imitation components are analyzed. The research results of individual samples of alcohol solutions and horilkas of different brands by the method of admittance spectroscopy were also analyzed. Based on this, methods for detecting falsification of horilka products by the method of admittance spectroscopy are proposed. The reactive component of the control object's ad is selected as an informative parameter. Two types of falsification are analyzed. The first is the falsification of the original horilka by replacing it with an aqueous-alcohol solution. The second - by replacing one brand of horilka with another. The analysis of the obtained results of researches of two types of objects on reactive components of their admittance in the frequency range $100 \mathrm{~Hz}-$ $100 \mathrm{kHz}$ showed the following: characteristic features of dependencies of reactive components on frequency are revealed; they have been found to have extreme values at different frequencies. The reactive component of the admittance of aqueous-alcohol solutions reaches an extreme value at the beginning of the range. The same component of the original horilkas of different brands has extreme values at the end of the range. Based on this, methods for prompt detection of falsifications are proposed.
\end{abstract}

Key words: Reactive component; Admittance; Alcohol; Quality; Falsification.

\section{Introduction}

Detection of falsification is carried out by organoleptic, chemical, and electrical methods. The organoleptic method is mostly ineffective, chemical methods require special tools and do not provide prompt control. Electrical methods ensure the objectivity and efficiency of control, as well as ease of implementation. An example of electrical methods is the method of impedance spectroscopy, which in recent decades has been used to control the parameters of various objects of non-electrical nature [1-7]. One such facility is alcohol, including horilka (a Ukrainian alcohol brand equivalent to whisky in Scotland). The mass appeared on the market of low-quality horilkas is due to individual counterfeiting of horilkas for the products of large manufacturers. Horilkas manufactured outside production control are not taken into account, as well as replacement in the process of transporting from producer to consumer by a counterfeit analog. The most common falsifications of horilkas are their replacement with an aqueousalcohol solution (alcohol and distilled water) or mismatch of the horilka. There is a method of determining the brands of horilka and cognac drinks [9]. An alternating electric current is applied to the sample of the investigated beverage and the ratio of the reactive and active components of the electrical conductivity of the beverage is determined from the alternating current frequency, then the minimum and maximum frequency dependence in its extremes. The alternating current is in the range from $0.1 \mathrm{~Hz}$ to $200 \mathrm{kHz}$, and the ratios at frequencies of $2 \mathrm{kHz}$ and $200 \mathrm{kHz}$ are determined. Comparing the obtained parameters with the reference values determine the brand of the drink.

\section{Shortcomings}

The disadvantage of the known electrical method is the wide range of frequencies within which measurements are made. It is necessary to measure many informative parameters, which complicates the technical means for implementing the method. The method does not raise the issue of detecting the fact of falsification of the drink.

\section{The Goal of the Work}

This work aims to develop a method for the rapid detection of horilka counterfeiting.

\section{Detection of Falsification by Impedance Spectroscopy \\ 4.1. Investigation of horilka products by impedance spectroscopy}

One of the electrical methods for detecting food adulteration is the imitation method (method of impedance or admittance spectroscopy). Researches of changes in the reactive and active components of admittance of the object under study have revealed classification features that can be used to quickly detect falsification of certain types of food products. To do this, it is necessary to compare the reactive components of the admittance of the object under study and the corresponding standard (base) samples. A characteristic feature of the dependence of the reactive component on the frequency of the test signal is the presence of an extreme value of the curve at individual frequencies. The 
difference between falsified horilka and basic liquid is that the extreme values of the reactive component are manifested at certain frequencies of different ranges.

\subsection{Analysis of the obtained results}

For researches, the three-electrode primary converter of a coaxial design and the measuring instrument of parameters of immittance BR 2827 are used. Active and reactive components of the admittance water-alcohol solution of different concentrations and horilka of different brands in the frequency range $100 \mathrm{~Hz}-$ $100 \mathrm{kHz}$ were measured. The results of researches [10] are demonstrated in Fig. 1, 2.

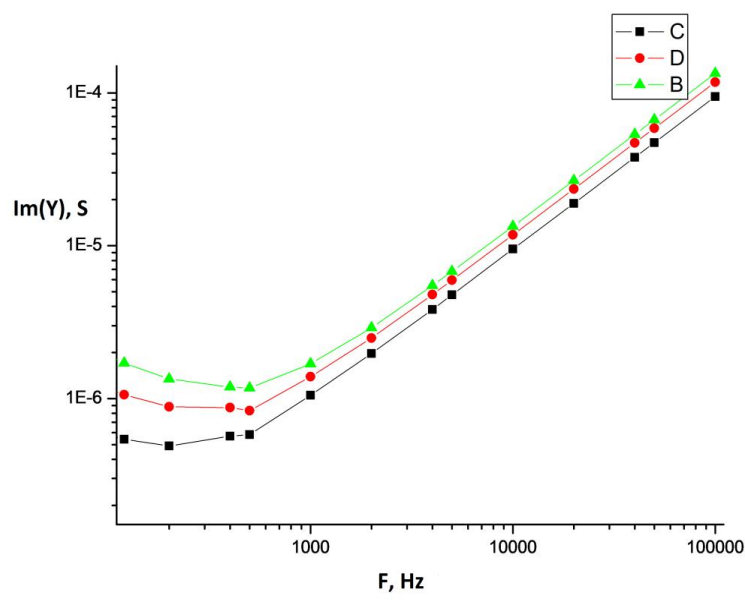

Fig. 1. Dependence of reactive components of admittances of alcohol solutions of different concentrations on frequency

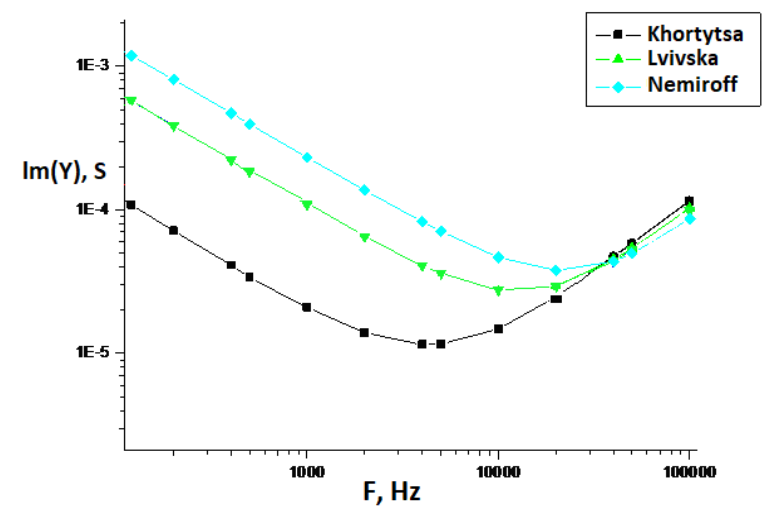

Fig. 2. Dependence of reactive components of admittances of horilkas of different brands on frequency

According to the results analysis of research (Fig. 1, 2): the difference in the nature of the reactive component of horilka is that it contains linear components (at the beginning of the range and the end) and a pronounced extreme value corresponding to a certain frequency. Each brand of horilka has its frequency at which this value is manifested. Similar extreme values are observed for alcohol solutions, but the frequency at which they are much lower than the frequency for horilkas. For the dilute aqueous-alcohol solution of different concentrations (water content in alcohol), the frequency is at the beginning of the range $100-300 \mathrm{~Hz}$ (Fig. 1), and for horilka in the range from $3 \mathrm{kHz}$ to $30 \mathrm{kHz}$ (Fig. 2).

\section{Ways of detecting falsification of horilka}

Based on the analysis of the obtained research results (Fig. 1, 2), an illustration of the implementation of methods of horilka falsification is presented.

Way 1. An illustration of the method of detecting counterfeit horilka with an aqueous-alcoholic solution is shown in Fig. 3, where the dependence of the reactive component of the admittance of the aqueous-alcoholic solution is curve $C$, and the original horilka is the curve $D$.

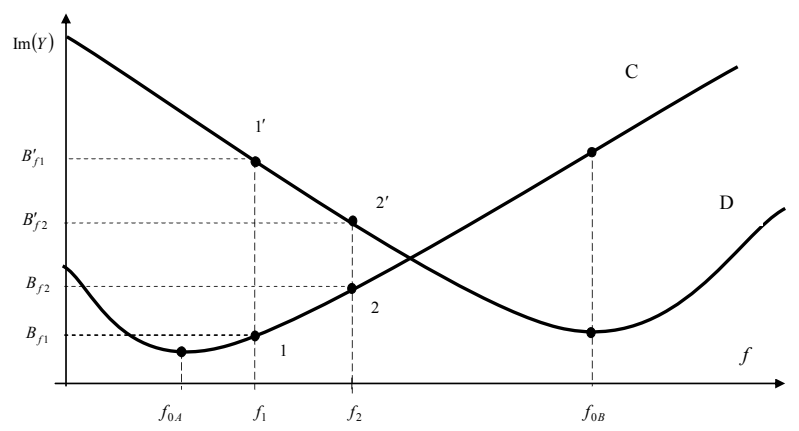

Fig. 3. Illustration of the way 1 for detecting counterfeit horilka

The frequencies $f_{O A}$ and $f_{O B}$, at which curves $C$ and $D$ have their extreme values, differ significantly (Fig. 3). For curve $C$, the reactive component in the frequency range $f_{O A}-f_{O B}$ is increasing with rising frequency, and for curve $D$ - decreases. In the specified frequency range, you must select the frequencies at which the value of curve $C$ increases and curve $D$ moves down. This can be considered an identifying feature of the detection of counterfeit horilka by replacing it with dilute alcohol.

To identify such an aqueous-alcohol solution (adulterated horilka), it is sufficient to measure the reactive component $\operatorname{lm}(Y)$ of the object's understudy admittance at two fixed frequencies $f_{1}$ and $f_{O B} f_{1}$ and $f_{2}$ (Fig. 3). According to the results of studies of wateralcohol solution of different concentrations, the frequency $f_{1}$ should be higher than $300 \mathrm{~Hz}$ (Fig. 1).

If the obtained value of the component at frequency $f_{1}$ is less than the value at frequency $f_{2}\left(B_{-} f_{1}<B_{f_{2}}\right)$, the horilka is falsified. If the measured value of the component at $\mathrm{f}_{1}$ is greater than the value at $f_{2}\left([B]_{-} f_{1}<[B] f_{2}\right)$ the controlled object is the original horilka.

Way 2. The other way of falsification of horilka due to its inconsistency with the brand indicated on the 
label is demonstrated in Fig. 4. Curves $M 1, M 2, M 3$ are the reactive components of different brands of horilka.

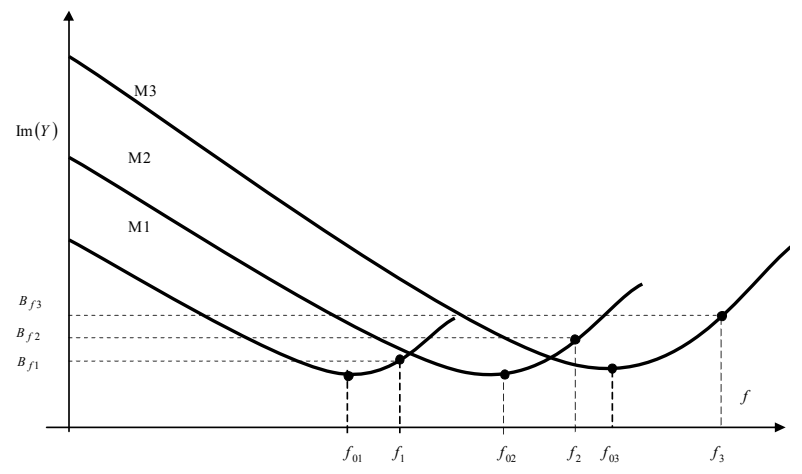

Fig. 4. Illustration of the way 2 for detecting falsification of horilka

These brands of horilka correspond to $f_{01}, f_{02}, f_{03}$ at which the reactive components acquire extreme values. To detect falsification of horilka due to noncompliance with the brand indicated on the label, it is necessary to check the reactive components at frequencies $f_{01}$ and $f_{1}$ for brand $M 1, f_{02}$ and $f_{2}$ for $M 2, f_{03}$ and $f_{3}$ for $M 3$ (Fig. 4) The values of components to $B_{01}$, $B_{02}, B_{03}$ and $B_{1}, B_{0}, B_{0}$ obtained at these frequencies must be compared. Under conditions $B_{01}<B_{1}, B_{02}<B_{2}$, $B_{03}<B_{3}$ horilkas correspond to the marks specified on labels, and under conditions $B_{01}>B_{1}, B_{02}>B_{2}, B_{03}>B_{3}$ horilkas are falsified by brand substitution.

\section{Conclusions}

Methods for detecting counterfeit horilka by admittance spectroscopy are proposed. To detect falsification of horilka with an alcohol solution, it is necessary to measure the reactive components at two frequencies in the range $100-300 \mathrm{~Hz}$, and by replacing the brand $-3-30 \mathrm{kHz}$. The obtained results must be compared in absolute terms.

\section{Gratitude}

The authors express their gratitude to the staff of the Department of Information and Measurement Technologies of the Lviv Polytechnic National University, Ukraine, for their assistance in the preparation of the article.

\section{Conflict of interest}

There are no financial, organizational, or other possible conflicts related to this work.

\section{References}

[1] A. Bauchot, F. Harker, W. Arnold, "The use of electrical impedance spectroscopy to assess the physiological condition of kiwifruit", Postharvest Biol. Tec., Vol.18, pp.9-18, Jan. 2000 [Online]. Available: DOI: 10.1016/S09255214(99)00056-3

[2] A. Chowdhury, T. Kanti Bera, D. Ghoshal, B. Chakraborty, "Electrical impedance variations in banana ripening: an analytical study with electrical impedance spectroscopy", J. Food Process. Eng., Vol.40, e12387, 2017. [Online]. Available: https://doi.org/10.1111/jfpe.12387

[3] M. Grossi, B. Riccò, "Electrical impedance spectroscopy (EIS) for biological analysis and food characterization", J. Sens. Sens. Syst., Vool.6, pp.303-325, 2017. [Online]. Available: DOI: 10.5194/jsss-6-303-2017

[4] E. Haverkort, P. Reijven, J. Binnekade, M. de van der Schueren, C. Earthman, D. Gouma, R. de Haan, "Bioelectrical impedance analysis to estimate body composition in surgical and oncological patients: a systematic review", European Journal of Clinical Nutrition, Vol. 69, pp. 3-13, 2015.

[5] A. Chowdhury, P. Singh, T. Kanti Bera, D. Ghoshal, B. Chakraborty, "Electrical impedance spectroscopy study of mandarin orange during ripening", J. Food Meas. Charact., Vol.1-11. [Online]. Available: https://ur.booksc.eu/ book/65152159/343622

[6] M. Grossi, A. Pompei, M. Lanzoni, R. Lazzarini, D. Matteuzzi, B. Riccò, "Total bacterial count in soft-frozen dairy products by impedance biosensor system", IEEE Sensors J., Vol.9, pp.1270-1276, 2009. [Online]. Available: https://www.academia.edu/14035472/Total_Bacterial_Count_i n_Soft_Frozen_Dairy_Products_by_Impedance_Biosensor_Sy stem

[7] C. Bhatt, J. Nagaraju, "Non-destructive method to estimate the moisture content in bread using multi-channel electrical impedance spectroscopy", IEEE Sensors Appl. Symp. New Orleans, LA, USA, 17-19 Febr. 2009. [Online]. Available: https://www.academia.edu/7725207/Non_destructive_method_ to_estimate_the_moisture_content_in_bread_using _ multi_channel_electrical_impedance_spectroscopy

[8] Patent of Ukraine №19620 IPC G01 N 33/14, G01 N R27 / 00, publ.15.12.2006, Bull. №12 